\section{Stabilization of proteins by glycosylation examined by NMR analysis of a fucosylated proteinase inhibitor}

\author{
Georges Mer, Hélène Hietter and \\ Jean-François Lefèvre
}

Nature Structural Biology 3, 45-53 (1996).

Figure 4 was misprinted. The version below is as it should have appeared in the January 1996 issue.

\section{$\operatorname{Arg} 18 \mathrm{NH} \varepsilon$ FU NF}

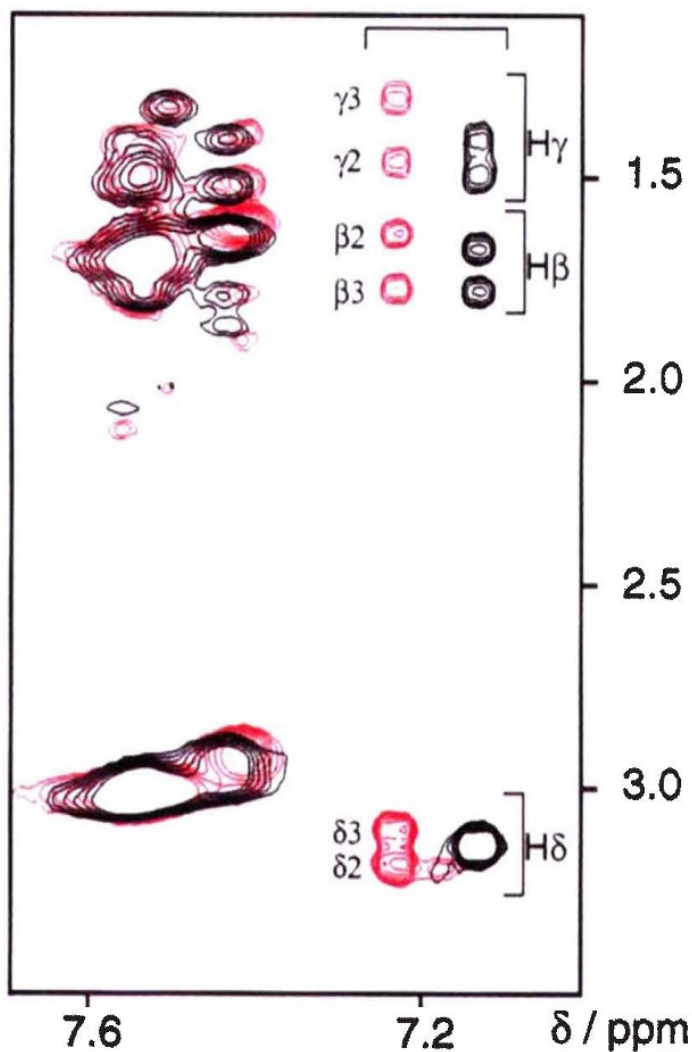

Fig. 4 Comparison of Arg 18 side-chain resonances in the nonfucosylated (shown in black) and fucosylated (shown in red) PMP-C inhibitors. Represented are portions of HOHA$\mathrm{HA}$ spectra acquired with spin-lock times of $80 \mathrm{~ms}$ at $30^{\circ} \mathrm{C}$ and $\mathrm{pH} 3$. Note that stereospecific assignments were only obtained for Arg 18 in the fucosylated inhibitor.

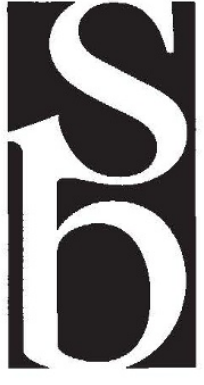

Nature Structural Biology is an international monthly journal publishing important advances in all fields relating to the structures of biological macromolecules as determined by X-ray crystallography, NMR, or electron diffraction, including improvements in the available methods for the determination of such structures, studies of biological mechanism or function in which the methods used are primarily structural, studies of macromolecular structure using molecular biological techniques, and methods for structure prediction from primary sequences. Editorial decisions concerning publication of manuscripts in NSB are to be made separately from those made at Nature. All editorial and production tasks are handled in Washington DC (see below).

Manuscripts are selected for publication according to editorial assessment of their general interest and suitability, and reports from independent referees. Receipt of all manuscripts will be acknowledged but those that are not suitable for review will be returned without delay. Contributors are welcome to suggest potential reviewers as well as informing the Editor of potential conflicts of interest. We urge clear writing to enhance the accessibility of manuscripts for readers not directly familiar with the field under investigation.

Authors of papers previously considered by Nature but ultimately not accepted (on editorial grounds or due to space constraints) are welcome to resubmit to Nature Structural Biology if they wish, where they will receive prompt further consideration. Publication of such papers may be offered without the need for further peer review, if the work is technically sound and meets the editorial requirements of Nature Structural Biology.

Following acceptance of their paper, contributors will receive galley proofs within a few weeks. Some editing is likely in order to enhance clarity and consistency. Contributors will receive a reprint order form with their proofs; reprint orders are processed from our New York office after the manuscript is published and payment received. Nature Structural Biology will not exact page charges.

\section{Format of Articles}

Manuscripts should generally follow the style and format of an article in Nature, with a Summary, Introduction, Results and Discussion and Methods.

TITLE should be simple and concise.

SUMMARY should be a brief, accessible summary of no more than 100 words should explain the goals, results and chief conclusions of the work, and must not include any references. Summaries for Correspondence are 20-30 words.

RESULTS should include short cross-headings to define the main aspects of the study and guide to the reader. We request that authors deposit sequence data in the databases, and provide an accession number in the paper.

METHODOLOGY will be a separate section placed at the end of the text, before the references (rather than being included in the figure legends). Papers should state that atomic coordinates, nucleic acid sequences, and protein sequences have been deposited in an appropriate databank and where possible give the entry name or accession number. Insights contain no Methods sections

REFERENCES are to be numbered sequentially as they appear in the text, followed by those in the figure legends and tables. Do not include any annotation. Full titles of papers are required. All authors should be listed unless there are six or more, in which case 'et al.' should be substituted. First and large page numbers in full must be included; references to books should include publisher, place and date. The list should include only papers published or in press; abstracts, papers submitted or in preparation and personal communications should be cited in the text. Where possible a reference to a review should be used; authors should attempt to keep references to a minimum. Full confirmation with our reference format will expedite handling of proofs. References in Correspondence omit the full title.

ORIGINAL ARTWORK (figures) should be submitted with the manuscript. Please provide 5 sets. Oversized artwork should be avoided. ACKNOWLEDGEMENTS should be brief.

SUBMISSION. An original and four copies of your manuscript should be sent to the Editor, Nature Structural Biology, 545 National Press Building, 529 14th St. NW, Washington DC 20045, USA. (phone: 202-626-2518; fax: 202-626-2528, e-mail: nsb@naturedc.com). Please provide current fax/phone numbers and e-mail addresses of the corresponding author on all submissions.

ON DISKETTE. Please note that we set all page proofs directly from computer discs provided by the authors. Any common PC or Mac word-processing package is compatible and preferable to a text/ASCII file. We cannot process Wordstar.

Editorial, suitability and production inquiries are welcome, as are proposals for Review or Progress articles, and should be directed to the Washington office. 